\title{
Decreased fertility in poor responder women is not related to oocyte morphological status
}

\author{
Marcílio Nichi', Rita de Cassia Sávio Figueira ${ }^{2}$, Daniela Paes de Almeida Ferreira Braga ${ }^{2,3}$, \\ Amanda Souza Setti ${ }^{3}$, Assumpto laconelli Jr' , Edson Borges $\mathrm{Jr}^{2-4}$
}

\author{
1Department of Animal Reproduction, Faculty of Veterinary Medicine, University of São \\ Paulo, Brazil \\ 2Fertility - Assisted Fertilization Center, São Paulo, Brazil \\ ${ }^{3}$ Sapientiae Institute - Educational and Research Center in Assisted Reproduction, São \\ Paulo, Brazil \\ 4Department of Gynecology and Obstetrics - Botucatu Medical School/UNESP, São Paulo, \\ Brazil
}

Submitted: 31 May 2010

Accepted: 24 June 2010

Arch Med Sci 2011; 7, 2: 315-320

DOI: 10.5114/aoms.2011.22084

Copyright @ 2011 Termedia \& Banach

\begin{abstract}
Introduction: In women showing impaired fertility, a decreased response to ovarian stimulation is a major problem, limiting the number of oocytes to be used for assisted reproduction techniques (ART). Despite the several definitions of poor response, it is still a matter of debate whether young poor responder patients also show a decrease in oocyte quality. The objective in this study was to investigate whether poor ovarian response to the superstimulation protocol is accompanied by impaired oocyte quality.

Material and methods: This study included 313 patients younger than 35 years old, undergoing intracytoplasmic sperm injection. Patients with four or fewer MII oocytes (poor-responder group, PR, $n=57$ ) were age-matched with normoresponder patients (NR, $n=256$ ).

Results: A higher rate of oocyte retrieval and a trend towards an increase in MII oocyte rate were observed in the NR group when compared to the PR group $(71.6 \pm 1.1 \%$ and $74.1 \pm 1.0 \%$ vs. $56.3 \pm 2.9 \%$ and $66.5 \pm 3.7 \% ; p<0.0001$ and $p=0.056$, respectively). A trend toward increased implantation rates was observed in the NR group when compared to the PR group (44 and $24.5 \pm 2.0 \%$ vs. 28.8 and $16.4 \pm 3.9 \% ; p=0.0305$ and $p=0.0651$, respectively).

Conclusions: Low response to ovarian stimulation is apparently not related to impaired oocyte quality. However, embryos produced from poor responder oocytes show impaired capacity to implant and to carry a pregnancy to term.
\end{abstract}

Key words: intracytoplasmic sperm injection, ovarian stimulation, oocyte retrieval.

\section{Introduction}

Approximately $40 \%$ to $50 \%$ of all infertility (i.e., no pregnancy after at least 1 year of unprotected intercourse [1]) occurs due to female factors. Ovulation dysfunction [2], scarring from inflammatory or infectious diseases [3], nutrient deficiencies [4], hormone imbalance [5], ovarian cysts [6], and transport system abnormality from the cervix through the fallopian tubes [7] are some of the causes of female infertility. Assisted reproductive technologies (ART) have been developed in order to overcome both female and male infertility. In women showing impaired fertility, a decreased

\author{
Corresponding author: \\ Edson Borges Jr, MD, PhD \\ Av. Brigadeiro Luis Antonio \\ 4545. Zip: 01401-002 \\ São Paulo - SP, Brazil \\ Phone: 5511 3018-8181 \\ E-mail: edson@fertility.com.br
}


response to ovarian stimulation, with incidence estimated to range from $9 \%$ to $24 \%$ [8-10], is a major problem, limiting the number of oocytes to be used for ART.

It is well known that ovarian poor response to stimulation protocols increases as women get older [10]. Furthermore, a decline in both oocyte quantity and quality is observed in older women (e.g., > 35 years old) [11]. On the other hand, in younger patients, a trend towards decreased fertility was observed when response to ovarian stimulation is impaired, despite no differences in ovarian reserve [12]. A hypothesis to explain such results is a decrease in oocyte quality. Despite the several definitions of poor response [12-16], it is still a matter of debate whether young poor responder patients also show a decrease in oocyte quality. The present study was performed in order to study whether ovarian response is related to decline in oocyte quality in women younger than 35 years old, avoiding the effect of age on oocyte quality.

\section{Material and methods}

\section{Patients}

Data of intracytoplasmic sperm injection (ICSI) cycles performed in 313 patients younger than 35 years old were included in this retrospective study. All cases of surgically retrieved sperm were excluded from the study. Patients were divided into those who produced four or less MII oocytes (poorresponder, PR group, $n=57$ ) after controlled ovarian stimulation (COS) and those in whom five or more oocytes were retrieved (normoresponder, NR group, $n=256)$. Written informed consent was obtained, in which patients agreed to share the outcomes of their own cycles for research purposes, and the study was approved by the local institutional review board.

\section{Ovarian stimulation and oocyte retrieval}

Controlled ovarian stimulation was achieved by long pituitary down-regulation using a gonadotropin-releasing hormone agonist (GnRHa, Lupron Kit ${ }^{T M}$, Abbott S.A Societé Française des Laboratoires, Paris, France) followed by ovarian stimulation with recombinant FSH (Gonal- ${ }^{\circledR}$, Serono, Geneve, Switzerland). The follicular dynamic was followed by ultrasound starting on day 4 of gonadotropin administration. When adequate follicular growth and serum oestradiol levels were observed, recombinant human chorionic gonadotrophin ( $r$-hCG, Ovidrel ${ }^{\mathrm{TM}}$, Serono, Geneve, Switzerland) was administered to trigger final follicular maturation. Oocytes were collected $35 \mathrm{~h}$ after hCG administration by transvaginal ultrasound ovum pick-up.

Oocytes were stored in human tubal cultured medium (HTF, Irvine Scientific, Santa Ana, USA) supplemented with 10\% Human Synthetic Albumin (HSA, Irvine Scientific, Santa Ana, USA) covered with oil (Ovoil ${ }^{\mathrm{TM}}$, Vitrolife, Kungsbacka, Sweden) at $37^{\circ} \mathrm{C}$ in $6 \% \mathrm{CO}_{2}$ for $5 \mathrm{~h}$, before cumulus cell removal. Cumulus cells were removed from the oocytes by placement of HEPES-buffered medium containing hyaluronidase (80 IU/ml Irvine Scientific, Santa Ana, USA). The remaining surrounding cumulus cells were then removed by gently pipetting with a handdrawn Pasteur pipette (Humagen Fertility Diagnostics, Charlottesville, USA).

\section{Preparation of oocytes and morphology assessment}

Retrieved oocytes were maintained in human tubal cultured medium (HTF, Irvine Scientific, Santa Ana, USA) supplemented with 10\% Human Synthetic Albumin (HSA, Irvine Scientific, Santa Ana, USA) covered with mineral oil (OvoilTM Vitrolife) for 2-3 h before cumulus cell removal. Surrounding cumulus cells were removed with exposure to a HEPES-buffered medium containing hyaluronidase (80 IU/ml, Irvine Scientific, Santa Ana, USA). The remaining cumulus cells were then mechanically removed by gently pipetting with a hand-drawn Pasteur pipette (Humagen Fertility Diagnostics, Charlottesville, USA). Oocyte morphology was assessed using an inverted Nikon Diaphot microscope (Eclipse TE 300; Nikon ${ }^{\circledR}$, Tokyo, Japan) with a Hoffmann modulation contrast system under $400 \times$ magnification, just before sperm injection (3-4 $\mathrm{h}$ after retrieval). The following extracytoplasmic (EC) and intracytoplasmic (IC) morphological abnormalities of the oocyte were recorded: (i) large perivitelline space size (EC), (ii) perivitelline space granularity (EC), (iii) fragmented first polar body (EC), (iv) increased cytoplasmic granularity (IC; dark centre or homogeneous), (v) smooth endoplasmic reticulum clusters (IC) and (vi) vacuoles in the ooplasm (IC). Normal oocytes showed clear cytoplasm with uniform texture and homogeneous fine granularity that did not contain inclusions, a small perivitelline space without granularity, and a single unfragmented first polar body (Veeck 1988).

\section{Intracytoplasmic sperm injection procedure}

Intracytoplasmic sperm injection was performed in MII oocytes according to the technique described by Palermo et al. [17]. Oocytes were transferred into the micro-injection dish, prepared with drops of HEPES-buffered HTF (Irvine Scientific, Santa Ana, USA) covered under oil and placed on a heated stage of an inverted microscope. Approximately $16 \mathrm{~h}$ after ICSI, fertilization was confirmed by the presence of two pronuclei and the extrusion of the second polar body. Embryos were kept in a $50 \mu \mathrm{l}$ 
drop of HTF medium supplemented with 10\% HAS under oil, in a humidified atmosphere of $5 \% \mathrm{CO}_{2}$ in air, at $37^{\circ} \mathrm{C}$, until transfer. Embryo transfer was performed on the third day of development.

\section{Clinical follow-up}

Serum $\beta$-hCG levels were assessed for the first time 12 days after replacement of the embryos. Clinical pregnancy was defined when a transvaginal ultrasound scan, performed 3-4 weeks after embryo transfer, revealed the presence of a gestational sac. To calculate the implantation rate, the number of gestational sacs was divided by the number of embryos transferred. Miscarriage was defined as spontaneous abortion before 20 weeks' gestation.

\section{Data analysis}

The two groups were compared with regard to: (i) age; (ii) oocyte yield (no. of retrieved oocytes/no. follicles); (iii) metaphase II oocyte rate (MII oocytes/total number of retrieved oocytes); (iv) percentage of extracytoplasmic (i.e., large perivitelline space size, perivitelline space granularity, and fragmented first polar body) and intracytoplasmic (i.e., increased cytoplasmic granularity, smooth endoplasmic reticulum clusters, and vacuoles in the ooplasm); (v) percentage of high quality embryos on the third day of development (no. of high quality embryos/no. of fertilized MII oocytes); (vi) pregnancy rate; (vii) implantation, and (viii) miscarriage rates.

High quality embryos were defined as those showing 6-8 cells on the third day of development, less than $15 \%$ fragmentation, symmetric blastomeres, absence of multinucleation, and absence of zona pellucida dysmorphism.

\section{Statistical analysis}

Results were expressed as mean \pm standard error for numeric variables, while proportions (\%) were used for categorical variables. Mean values were compared by Student's $t$ parametric test or Mann-Whitney non-parametric test, according to
Gaussian distribution and variance homogeneity. Proportions were compared by the $\chi^{2}$ or Fisher exact test, only when the expected frequency was five or less. Results were considered to be significant at the $5 \%$ critical level $(p<0.05)$. Data analysis was carried out using SAS System for Windows.

\section{Results}

Poor responder patients showed higher age than the normal responders (NR: $30.4 \pm 0.2$ vs. PR: 31.7 $\pm 0.3, p=0.0324$ ). Furthermore, despite similar duration of gonadotrophin stimulation in the two groups, significantly increased doses of gonadotrophins were used in the PR group. Oestradiol concentration on the day of hCG and oocyte yield were significantly lower in the PR group (Table I).

Oocyte retrieval rate was significantly lower for the PR group than for the NR group ( $56.3 \pm 2.9$ vs. $71.6 \pm 1.1$, respectively, $p<0.0001$, Table I). Similarly, a trend for a difference was found when comparing MIl oocytes rates among groups (PR: $66.5 \pm 0.3 .7$ vs. $74.1 \pm 1.0, p=0.056$ ).

Regarding oocyte morphological abnormalities, the PR group showed a higher percentage of oocytes showing a large perivitelline space when compared to the NR group ( $31.8 \pm 4.7$ vs. $21.5 \pm 1.8 \%$, respectively; $p=0.0202$ ). No differences were found between groups regarding the remaining oocyte morphological abnormalities (Table II).

Despite the fact that no differences were found among groups in the rate of high quality embryos (PR: $49.7 \pm 5.3$ vs. NR: $50.3 \pm 1.9, p=0.9135$ ), the pregnancy rate was significantly different (PR: 28.8 vs. NR: $44 \%, p=0.0305$, Table III). A trend towards a higher implantation rate was found in normal responder patients when compared to poor responders ( $24.5 \pm 2.0$ vs. $16.4 \pm 3.9$, respectively, $p=0.0651$, Table III).

\section{Discussion}

There is no universal definition of poor responder. Numbers of mature follicles noted on

Table I. Stimulation cycle characteristics in a group of poor responder patients younger than 35 years (PR group) compared with age-matched normoresponders (NR group)

\begin{tabular}{|lccc|}
\hline & \multicolumn{2}{c|}{ Study group } \\
& \multicolumn{2}{c|}{ Value of $p$} \\
\cline { 2 - 4 } & PR $(n=57)$ & $30.4 \pm 0.2$ & 0.0324 \\
\hline Age [years] & $31.7 \pm 0.3$ & $2023.8 \pm 43.8$ & 0.0211 \\
\hline Total gonadotrophin dose [IU] & $2318.1 \pm 117.4$ & $1022.74 \pm 95.9$ & 0.0020 \\
\hline Oestradiol on day of hCG [pg/ml] & $388.7 \pm 76.8$ & $71.6 \pm 1.1$ & $<0.0001$ \\
\hline No. of retrieved oocytes/No. of follicles [\%] & $56.3 \pm 2.9$ & $74.1 \pm 1.0$ & 0.0546 \\
\hline Mll oocytes/total number of retrieved oocytes [\%] & $66.5 \pm 3.7$ & & \\
\hline
\end{tabular}

Values expressed as mean \pm SEM, unless otherwise noted. Student's t-test 
Table II. Effect of ovarian response (poor vs. normal) on oocyte morphology of patients younger than 35 years

\begin{tabular}{|lccc|}
\hline & \multicolumn{2}{c}{ Study group } & \multirow{2}{*}{ Value of $p$} \\
\cline { 2 - 3 } & $\mathrm{PR}(n=57)$ & $\mathrm{NR}(n=256)$ & \\
\hline Large perivitelline space size & $31.8 \pm 4.7$ & $21.5 \pm 1.8$ & 0.0202 \\
\hline Perivitelline space granularity & $33.7 \pm 4.7$ & $36.1 \pm 2.2$ & 0.6423 \\
\hline Fragmented first polar body & $26.8 \pm 4.3$ & $31.7 \pm 2.0$ & 0.2864 \\
\hline Increased cytoplasmic granularity & $61.3 \pm 5.4$ & $66.1 \pm 2.5$ & 0.3975 \\
\hline Smooth endoplasmic reticulum clusters & $8.0 \pm 3.0$ & $6.8 \pm 1.2$ & 0.7025 \\
\hline Vacuoles in the ooplasm & $3.1 \pm 1.5$ & $3.3 \pm 0.6$ & 0.8617 \\
\hline
\end{tabular}

Values expressed as mean \pm SEM, unless otherwise noted, Student's t-test

Table III. Intracytoplasmic sperm injection cycle outcomes in a group of poor responder patients younger than 35 years (PR group) compared with age-matched normoresponders

\begin{tabular}{|lccc|}
\hline & \multicolumn{2}{c|}{ Study group } & \multicolumn{2}{c|}{ Value of $p$} \\
\cline { 2 - 4 } & PR $(n=57)$ & $50.3 \pm 1.9$ & $0.9135^{\mathrm{a}}$ \\
\hline Rate of high quality embryos & $49.7 \pm 5.3$ & 44 & $0.0305^{\mathrm{b}}$ \\
\hline Pregnancy rate & 28.8 & $256)$ \\
\hline Implantation rate & $16.4 \pm 3.9$ & $0.0651^{\mathrm{a}}$ \\
\hline Abortion rate & 10.1 & 6.2 & $0.2933^{\mathrm{b}}$ \\
\hline
\end{tabular}

Values are percentages. ${ }^{a}$ Student's $t$-test, ${ }^{b}$ Fisher exact test

ultrasound [13], high basal FSH levels in the early follicular phase [16], cumulative dose or days of gonadotropin requirements in a prior cycle [18], and numbers of mature oocytes obtained [19] have been used. In the present experiment, a reduced number of MII oocytes (e.g., less than 5) was used to define poor response to ovarian stimulation. Hellberg et al. [20], verified that women showing less than four retrieved oocytes showed a significant decrease in pregnancy rate following IVF. Furthermore, according to Arnot et al. [21], IVF success is significantly related to the number of oocytes retrieved. Therefore, women showing a limited number of oocytes following ovum pick-up, especially MII oocytes, present impaired embryo production and, therefore, decreased chances of pregnancy.

Studies indicate the deleterious influence of age on oocyte yield and quality. In fact, older women (above 35 years old) present a reduction in follicular diameter when compared to younger women, suggesting that only larger follicles are generally recruited in the beginning of reproductive life, and as women get older the remaining follicles show a decrease not only in diameter but in quality as well [22]. The limit of 35 years old has been widely used in studies concerning the effect of age on assisted reproduction outcome. In the present study, even in women younger than 35 years old, women in the poor responder group were statistically older than women in the normal responder group, indicating that the age of 35 years may not be ideal to study the influence of age on ovarian response to stimulation.

Besides showing a lower number of oocytes retrieved, the rates of oocyte retrieval and MII oocyte retrieved was also lower in poor responder women. Similar results were obtained by Younis et al. [23]; $14 \%$ of patients showed a decrease in oocyte retrieval rate. A hypothesis to explain such results would be the presence and aspiration of a significant number of small follicles, which may not contain eggs capable of collection, such as those observed in polycystic ovary syndrome [24]. Further studies are necessary to confirm this hypothesis.

In order to be considered morphologically normal, an MII oocyte should present a round, even shape, cytoplasm with homogeneous granularity, a small perivitelline space with no debris, and a transparent zona pellucida (ZP) [25]. The detailed analysis of oocyte morphological status during the IVF cycle is impaired by the surrounding cumulus cells. On the other hand, cumulus cells are removed when employing ICSI allowing the visualization of morphological structure and nuclear maturity. Furthermore, the impact of oocyte morphology on fertility when using IVF/ICSI is a matter of debate [26]. In the present experiment, the group of poor responder patients showed increased percentages of large perivitelline space. This was accompanied by a significant decrease in pregnancy rate and a trend towards impaired embryo implantation. This 
morphological abnormality may affect oocyte survival [27, 28] and fertilization rate after insemination by ICSI [29]. This feature seems to reflect an over-maturity of the oocytes at the moment of ICSI [30]. However, different studies have failed to find a correlation between size and shape of the perivitelline space and fertilization rate and embryo development [31, 32]. The negative effect of this morphology may be related to its extent and to the simultaneous presence of other abnormalities, such as non-nucleated fragments. However, in the present study when scores of abnormalities were considered (i.e., score 1: one abnormality; score 2: two abnormalities; score 3: three abnormalities), no differences were found between groups (data not shown), indicating that poor capacity of embryos produced from poor responder patients is not related to the number of morphological abnormalities found in the oocytes. However, further studies are necessary to evaluate the competence of embryos produced from oocytes showing a large perivitelline space.

Despite the higher number of available embryos in the NR group, which may have influenced pregnancy rates by limiting the selection for transfer, the trend towards a higher implantation rate indicates that embryos collected from poor responder patients may present impaired biological capacity.

In conclusion, our results suggest that although apparently high quality embryos may be produced in women showing poor response to ovarian stimulation, care must be taken regarding the possibility of pregnancy being carried to term in such patients.

\section{References}

1. WHO, Infertility: a tabulation of available data on prevalence of primary and secondary infertility. Programme on Maternal and Child Health and Family Planning, Division of Family Health. World Health Organization, Geneva 1991.

2. Devoto L, et al. Human corpus luteum physiology and the luteal-phase dysfunction associated with ovarian stimulation. Reprod Biomed Online 2009; 18 Suppl 2: 19-24.

3. Hillis SD, Joesoef R, Marchbanks PA, et al. Delayed care of pelvic inflammatory disease as a risk factor for impaired fertility. Am J Obstet Gynecol 1993; 168: 1503-9.

4. Tata LJ, Card TR, Logan RF, et al. Fertility and pregnancyrelated events in women with celiac disease: a population-based cohort study. Gastroenterology 2005; 128: 849-55.

5. Dierich A, Sairam MR, Monaco L, et al. Impairing folliclestimulating hormone (FSH) signaling in vivo: targeted disruption of the FSH receptor leads to aberrant gametogenesis and hormonal imbalance. Proc Natl Acad Sci U S A 1998; 95: 13612-7.

6. Benaglia L, Somigliana E, Vercellini P, Abbiati A, Ragni G, Fedele L. Endometriotic ovarian cysts negatively affect the rate of spontaneous ovulation. Hum Reprod 2009; 24: 2183-6.

7. McQueen D, McKillop JH, Gray HW, Bessent RG, Black WP. Investigation of tubal infertility by radionuclide migration. Hum Reprod 1991; 6: 529-32.

8. Ben-Rafael Z, Bider D, Dan U, et al. Combined gonadotropin releasing hormone agonist/human menopausal gonadotropin therapy (GnRH-a/hMG) in normal, high, and poor responders to hMG. J In Vitro Fert Embryo Transf 1991; 8: 33-6.

9. Jenkins JM, Davies DW, Devonport $\mathrm{H}$, et al. Comparison of 'poor' responders with 'good' responders using a standard buserelin/human menopausal gonadotrophin regime for in-vitro fertilization. Hum Reprod 1991; 6: 918-21.

10. Surrey ES, Schoolcraft WB. Evaluating strategies for improving ovarian response of the poor responder undergoing assisted reproductive techniques. Fertil Steril 2000; 73: 667-76.

11. te Velde ER, Pearson PL. The variability of female reproductive ageing. Hum Reprod Update 2002; 8: 141-54.

12. Figueira Rde C, Braga DP, Nichi M, et al. Poor ovarian response in patients younger than 35 years: is it also a qualitative decline in ovarian function? Hum Fertil (Camb) 2009; 12: 160-5.

13. Busacca M, Fusi FM, Brigante C, et al. Use of growth hormone-releasing factor in ovulation induction in poor responders. J Reprod Med 1996; 41: 699-703.

14. Feldberg D, Farhi J, Ashkenazi J, et al. Minidose gonadotropin-releasing hormone agonist is the treatment of choice in poor responders with high follicle-stimulating hormone levels. Fertil Steril 1994; 62: 343-6.

15. Hughes SM, et al. A double-blind cross-over controlled study to evaluate the effect of human biosynthetic growth hormone on ovarian stimulation in previous poor responders to in-vitro fertilization. Hum Reprod 1994; 9: 13-8.

16. Karande V, Morris R, Rinehart J, et al. Limited success using the "flare" protocol in poor responders in cycles with low basal follicle-stimulating hormone levels during in vitro fertilization. Fertil Steril 1997; 67: 900-3.

17. Palermo G, Joris H, Devroey P, Van Steirteghem AC. Pregnancies after intracytoplasmic injection of single spermatozoon into an oocyte. Lancet 1992; 340: 17-8.

18. Hofmann GE, Toner JP, Muasher SJ, et al. High-dose follicle-stimulating hormone (FSH) ovarian stimulation in low-responder patients for in vitro fertilization. J In Vitro Fert Embryo Transf 1989; 6: 285-9.

19. Rombauts L, Suikkari AM, MacLachlan V, Trounson AO, Healy DL. Recruitment of follicles by recombinant human follicle-stimulating hormone commencing in the luteal phase of the ovarian cycle. Fertil Steril 1998; 69: 665-9.

20. Hellberg D, Waldenstrom U, Nilsson S. Defining a poor responder in in vitro fertilization. Fertil Steril 2004; 82: 488-90.

21. Arnot AM, Vandekerckhove P, DeBono MA, Rutherford AJ. Follicular volume and number during in-vitro fertilization: association with oocyte developmental capacity and pregnancy rate. Hum Reprod 1995; 10: 256-61.

22. Westergaard CG, Byskov AG, Andersen CY. Morphometric characteristics of the primordial to primary follicle transition in the human ovary in relation to age. Hum Reprod 2007; 22: 2225-31.

23. Younis JS, Skournik A, Radin O, Haddad S, Bar-Ami S, Ben-Ami M. Poor oocyte retrieval is a manifestation of low ovarian reserve. Fertil Steril 2005; 83: 504-7.

24. Adams J, Polson DW, Abdulwahid N, et al. Multifollicular ovaries: clinical and endocrine features and response to 
pulsatile gonadotropin releasing hormone. Lancet 1985; 2: 1375-9.

25. VeeckLL. Oocyte assessment and biological performance. Ann N Y Acad Sci 1988; 541: 259-74.

26. Esfandiari N, Ryan EA, Gotlieb L, Casper RF. Successful pregnancy following transfer of embryos from oocytes with abnormal zona pellucida and cytoplasm morphology. Reprod Biomed Online 2005; 11: 620-3.

27. Ebner T, Yaman C, Moser M, Sommergruber M, Jesacher K, Tews $\mathrm{G}$. A prospective study on oocyte survival rate after ICSI: influence of injection technique and morphological features. J Assist Reprod Genet 2001; 18: 623-8.

28. Plachot M, Selva J, Wolf JP, Bastit P, de Mouzon J. Consequences of oocyte dysmorphy on the fertilization rate and embryo development after intracytoplasmic sperm injection. A prospective multicenter study. Gynecol Obstet Fertil 2002; 30: 772-9.

29. Rienzi L, Ubaldi FM, lacobelli M, et al. Significance of metaphase II human oocyte morphology on ICSI outcome. Fertil Steril 2008; 90: 1692-700.

30. Mikkelsen AL, Lindenberg S. Morphology of in-vitro matured oocytes: impact on fertility potential and embryo quality. Hum Reprod 2001; 16: 1714-8.

31. Balaban B, Urman B. Effect of oocyte morphology on embryo development and implantation. Reprod Biomed Online 2006; 12: 608-15.

32. Balaban B, Urman B, Sertac A, et al. Oocyte morphology does not affect fertilization rate, embryo quality and implantation rate after intracytoplasmic sperm injection. Hum Reprod 1998; 13: 3431-3. 